\title{
Application of Experiential Design for Framing Gastroretentive Microsponges of Glipizide: Screening of Critical Variables by Plackett-Burman Design and Optimization by Box-Behnken Design
}

\author{
Meenakshi Bhavesh Patel ${ }^{1, *}$, Farhatjahan Shaikh², Vandana B. Patel ${ }^{2}$, Naazneen Surti ${ }^{2}$ \\ ${ }^{1}$ Krishna School of Pharmacy \& Research, Drs. Kiran \& Pallavi Patel Global University (KPGU), Krishna Edu Campus, Varnama, \\ Vadodara, Gujarat, INDIA. \\ 'Babaria Institute of Pharmacy, BITS Edu Campus, Varnama, Vadodara, Affiliated to Gujarat Technological University, Ahmedabad, \\ Gujarat, INDIA.
}

\begin{abstract}
Introduction: Microsponges provides a proficient drug delivery system for specific delivery in the upper gastrointestinal tract with high drug loading capability. But the formulation is affected by numerous process and design related factors. The intention of the current research work was to formulate and optimize the floating gastroretentive microsponges of glipizide, an antidiabetic drug, with minimum number of experiments, by applying an appropriate experimental design. Methods: The decisive factors affecting formulation were screened by Plackett-Burman design and the final optimization were performed by applying Box-Behnken design. In Plackett-Burman design, Pareto ranking analyses indicated that polymer concentration, stirring speed and temperature significantly affected the formulation of microsponges. The influence of these independent variables was checked on the entrapment efficiency, buoyancy and cumulative drug release $(12 \mathrm{hr})$ of the formulation by Box-Behnken design. Results: The results of Box-Behnken design showed that after applying the desirability criteria and looking into overlay plots, formulation GBB-8 with medium drug-polymer ratio and maximum level of the other two variables was found to be optimum with desirability near 1 . The formulation gave entrapment efficiency as $90.81 \%$, buoyancy as $92.3 \%$ and $\mathrm{CDR}_{12}$ as $92.3 \%$. Radiographic studies conducted on albino rabbits, indicated the presence of the microsponges in the stomach for $12 \mathrm{hr}$. Conclusion: It could be concluded that application of experimental design is helpful tool for the development of floating microsponges of glipizide. The prepared formulation can offer the sustained release of the drug at its site of absorption which may provide the better control of the diabetes due to less fluctuation in plasma drug concentration.
\end{abstract}

Key words: Glipizide, Microsponges, Plackett-Burman design, Box-Behnken design, Antidiabetic.

\section{INTRODUCTION}

Microsponges were not explored for floating gastro retentive system until Arya et al. Developed targeted floating curcumin microsponges for improved site specific absorption for gastric cancer. ${ }^{1}$ These curcumin microsponges proved that they have floating ability and can be used for the gastroretention of the drugs. Hence, floating microsponges are the novel means of preparing the gastroretentive formulations for antidiabetic drugs, which are needed to be present in the upper division of GIT for its better therapeutic action.
Submission Date: 23-01-2020; Revision Date: 07-07-2020; Accepted Date: 08-10-2021

DOI: 10.5530/ijper.55.4.197 Correspondence: Dr. Meenakshi Bhavesh Patel,

Krishna School of Pharmacy \& Research, Drs. Kiran \& Pallavi Patel Global University (KPGU), Krishna Edu. Campus, Varnama, Vadodara, Gujarat, INDIA. Phone: +91-9924431093, Email-meenakshi_raina@ rediffmail.com

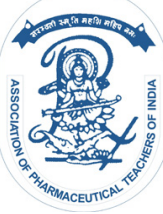

www.ijper.org 
Although many gastroretentive formulations of glipizide (GLP) have been developed but no work has been reported on its floating microsponges. ${ }^{2}$ Hence, the present research was intended for developing the floating microsponges of GLP with more loading capacity and better release of the drug in stomach. Furthermore, the formulation is supposed to provide sustained glucose lowering effect and improved diabetic condition as compared to immediate release of glipizide to achieve better treatment of the disease.

The literature review suggested that GLP is weakly acidic in nature with $\mathrm{pKa}$ value equal to 5.9 , which means that the drugs remains unionized at acidic $\mathrm{pH} .{ }^{3,4}$ The unionization is the prerequisite for the drugs to get absorbed by passive diffusion mechanism. Hence, the gastroretentive dosage form of GLP is desired. The elimination half-life of GLP is 2-4 h, which demands frequent administration of drug, to maintain its level in the body for extended period of time. Gastroretentive dosage form overcomes that demerit by releasing the drug continuously in the upper part of gastrointestinal tract, thereby achieving the better control of plasma glucose level. ${ }^{5,6}$ Gastroretentive floating Microsponges of GLP were prepared by quasi-emulsion solvent diffusion method. The Plackett-Burman design was employed as the screening technique to determine the most significant factors that affected the formulation of microsponges of GLP using Design-Expert ${ }^{\circledR}$ software. Plackett-Burman (PB) designs are used for screening experiments because, in a $\mathrm{PB}$ design, main effects are, in general, heavily confounded with two-factor interactions. ${ }^{7}$ The PB design in 12 runs, for example, may be used for an experiment containing up to 11 factors. This designs is very useful for economically detecting large main effects, assuming all interactions are negligible when compared with the few important main effects. ${ }^{8,9}$ Hence, this statistical tools was used for selecting the most important variables that can affect the formulation of microsponges.

The final optimization of glipizide floating microsponges was done by applying Box-Behnken design. ${ }^{10}$ The Box-Behnken design is an independent quadratic design in that it does not contain an embedded factorial or fractional factorial design. In this design the treatment combinations are at the midpoints of edges of the process space and at the center. These designs are rotatable (or near rotatable) and require 3 levels of each factor. The designs have limited capability for orthogonal blocking compared to the central composite designs. This is a very useful for developing a formulation as it requires less experimentation and provides assessments of the relative significance of different variables. ${ }^{11}$

\section{MATERIALS AND METHODS}

\section{Materials}

Glipizide was obtained as a gift sample from Micro labs, Mumbai. Ethyl cellulose was procured as a gift sample from Colorcon Asia Pvt Ltd, Goa. Polyvinyl alcohol was purchased from Sulab, Suvidhinath Laboratories, Vadodara. Triethylcitrate was obtained from SD Fine Ltd., Mumbai. Ethanol was procured from Ambica Enterprise, Vadodara and dichloromethane was obtained from Chemdyes Corporation, Rajkot.

\section{Preparation of GLP Microsponges}

Microsponges were prepared by quasi-emulsion solvent diffusion method using an external phase containing distilled water and polyvinyl alcohol (PVA). ${ }^{12,13}$ The internal phase was prepared by adding ethyl cellulose in the organic solvent system consisting of ethyl alcohol and Dichloromethane (DCM) as a bridging liquid. ${ }^{14}$ To this organic phase, Glipizide was added and dissolved completely. At last triethylcitrate (TEC) which was added in organic phase to facilitate the plasticity. Then, the inner phase was poured into outer phase with stirring. After emulsification, the mixture was continuously stirred on mechanical stirrer, (REMI Equipments Pvt. Ltd.), for a specified time and at a specific temperature. Once the process was finished, the product was immediately filtered to separate the microsponges. The product was washed and dried at room temperature for $24 \mathrm{~h}$.

\section{Screening of critical factors influencing microsponges using Plackett and Burman design}

The literature review suggested that there are many formulation and process related factors that can affect the formulation of microsponges and the influence of critical formulation and process parameter can be found by applying appropriate statistical design. ${ }^{15}$ Hence, in order to formulate the gastroretentive floating microsponges of glipizide, most significant factors were selected by implementing the Plackett-Burman design. PB design has been earlier applied for the formulation of gastroretentive therapeutic system of atenolol. ${ }^{16}$ In total, seven different factors were selected with their respective ranges for the formulation of floating microsponges, to evaluate their effect on the chosen responses, as shown in Table 1. The parameter level selection was done based on preliminary experiments and literature survey. Design-Expert software (trial version 9.0.6, Stat-Ease) was used to apply Plackett-Burman design 
Table 1: Formulation of microsponges using Plackett-Burman design.

A: Factors and Their Levels Used in Plackett-Burman Design*

\begin{tabular}{|c|c|c|c|c|c|c|c|}
\hline \multicolumn{1}{|c|}{ A: Factors and Their Levels Used in Plackett-Burman Design* } \\
\hline Factor codes & $\mathrm{X}_{1}$ & $\mathrm{X}_{2}$ & $\mathrm{X}_{3}$ & $\mathrm{X}_{4}$ & $\mathrm{X}_{5}$ & $\mathrm{X}_{6}$ & $\mathrm{X}_{7}$ \\
\hline Low level (-1) & 30 & $1: 2$ & 700 & 10 & 0.5 & $1: 3$ & 3 \\
\hline High Level (1) & 40 & $1: 4$ & 1500 & 20 & 1 & $1: 5$ & 4 \\
\hline
\end{tabular}

\begin{tabular}{|c|c|c|c|c|c|c|c|c|c|}
\hline Run & $\mathbf{X}_{1}$ & $\mathbf{X}_{2}$ & $\mathbf{X}_{3}$ & $\mathbf{X}_{\mathbf{4}}$ & $\mathbf{X}_{\mathbf{5}}$ & $\mathbf{X}_{6}$ & $\mathbf{X}_{\mathbf{7}}$ & $\begin{array}{c}\text { \% Yield } \\
\begin{array}{c}\text { Entrapment } \\
\text { Efficiency }\end{array}\end{array}$ \\
\hline 1 & 1 & -1 & 1 & -1 & -1 & -1 & 1 & 73.53 & $70.36 \pm 3.8$ \\
\hline 2 & 1 & 1 & -1 & 1 & -1 & -1 & -1 & 85.87 & $82.7 \pm 2.4$ \\
\hline 3 & -1 & 1 & 1 & -1 & 1 & -1 & -1 & 82.24 & $81.32 \pm 2.3$ \\
\hline 4 & 1 & -1 & 1 & 1 & -1 & 1 & -1 & 75.68 & $86.34 \pm 1.8$ \\
\hline 5 & 1 & 1 & -1 & 1 & 1 & -1 & 1 & 80.4 & $79.21 \pm 4.2$ \\
\hline 6 & 1 & 1 & 1 & -1 & 1 & 1 & -1 & 85.2 & $85.8 \pm 3.8$ \\
\hline 7 & -1 & 1 & 1 & 1 & -1 & 1 & 1 & 93.73 & $82.25 \pm 2.6$ \\
\hline 8 & -1 & -1 & 1 & 1 & 1 & -1 & 1 & 68.8 & $63.81 \pm 1.9$ \\
\hline 9 & -1 & -1 & -1 & 1 & 1 & 1 & -1 & 55.34 & $67.2 \pm 5.1$ \\
\hline 10 & 1 & -1 & -1 & -1 & 1 & 1 & 1 & 58.1 & $72.4 \pm 4.3$ \\
\hline 11 & -1 & 1 & -1 & -1 & -1 & 1 & 1 & 73.8 & $69.7 \pm 3.5$ \\
\hline 12 & -1 & -1 & -1 & -1 & -1 & -1 & -1 & 53.3 & $52.7 \pm 3.5$ \\
\hline
\end{tabular}

* $\mathrm{X}_{1}$ : Temperature $\left({ }^{\circ} \mathrm{C}\right) ; \mathrm{X}_{2}$ : Drug-Polymer Ratio; $\mathrm{X}_{3}$ : Speed of Agitation (rpm); $\mathrm{X}_{4}$ : Volume of TEC (\% of polymer concentration); $\mathrm{X}_{5}$ : PVP concentration $(\%) ; \mathrm{X}_{6}$ : DCM:Ethanol concentration; $X_{7}:$ Stirring time (hrs)

with 12 runs (Table 1). The dependent variable were percentage yield and percentage entrapment efficiency.

\section{Optimization of GLP Microsponges by Box- Behnken Design}

Plackett-Burman (PB) screening design helped in the identification of crucial factors, affecting the formulation of GLP microsponges. Taking those factors into the consideration, a response surface method, three-factor, three-level Box-Behnken design was applied for the final optimization of floating glipizide micro sponge. ${ }^{15}$ The low and high levels of factors were directly adopted from the previous Plackett-Burman design and the medium levels were set as the midpoint of low and high levels (Table 2). The drug polymer ratio, stirring speed and temperature were taken as independent factors. Whereas, $\%$ entrapment efficiency, $\%$ buoyancy and \% CDR12h were considered as dependent responses. Design-Expert ${ }^{\circledR}$ software (trial version 9.0.6, Stat-Ease) was used to apply the design and total 15 runs were formulated. Box-Behnken Design matrix with response value is given in Table 2. In addition, four other factors, which were evaluated in Plackett- Burman design, were set at a fixed level (PVA-0.5\%, TEC-20\%of polymer concentration, DCM:Ethanol-1:3 and stirring time-3hrs), in Box-Behnken design as their effects on the response variables seemed statistically insignificant as per the results obtained from Plackett-Burman design.

\section{Evaluation of Microsponges Entrapment efficiency}

Glipizide loaded microsponges theoretically equivalent to $10 \mathrm{mg}$ of glipizide were weighed, crushed and extracted with $5 \mathrm{ml}$ of methanol by vortexing. The sample was centrifuged at $2000 \mathrm{rpm}$ for $10 \mathrm{~min}$, filtered and assayed spectrophotometrically at $276 \mathrm{~nm}$ after appropriate dilution with methanol. Entrapment efficiency was determined by dividing the practically entrapped amount of drug to the total amount of drug. ${ }^{17}$

\section{Product Yield, Particle size and Measurement of Bulk Density}

Product yield of microsponges was calculated by dividing the weight of microsponges to total amount of drug and excipients taken for the preparation of microsponges. ${ }^{18}$ The particle size was measured by optical microscopy. The sample was placed on a slide and viewed under the trinocular microscope (Carl Zeiss). The mean particle size was calculated by measuring more than 300 microsponges. All the batches of prepared microsponges were placed in graduated measuring cylinder to find its bulk density using following formula: 


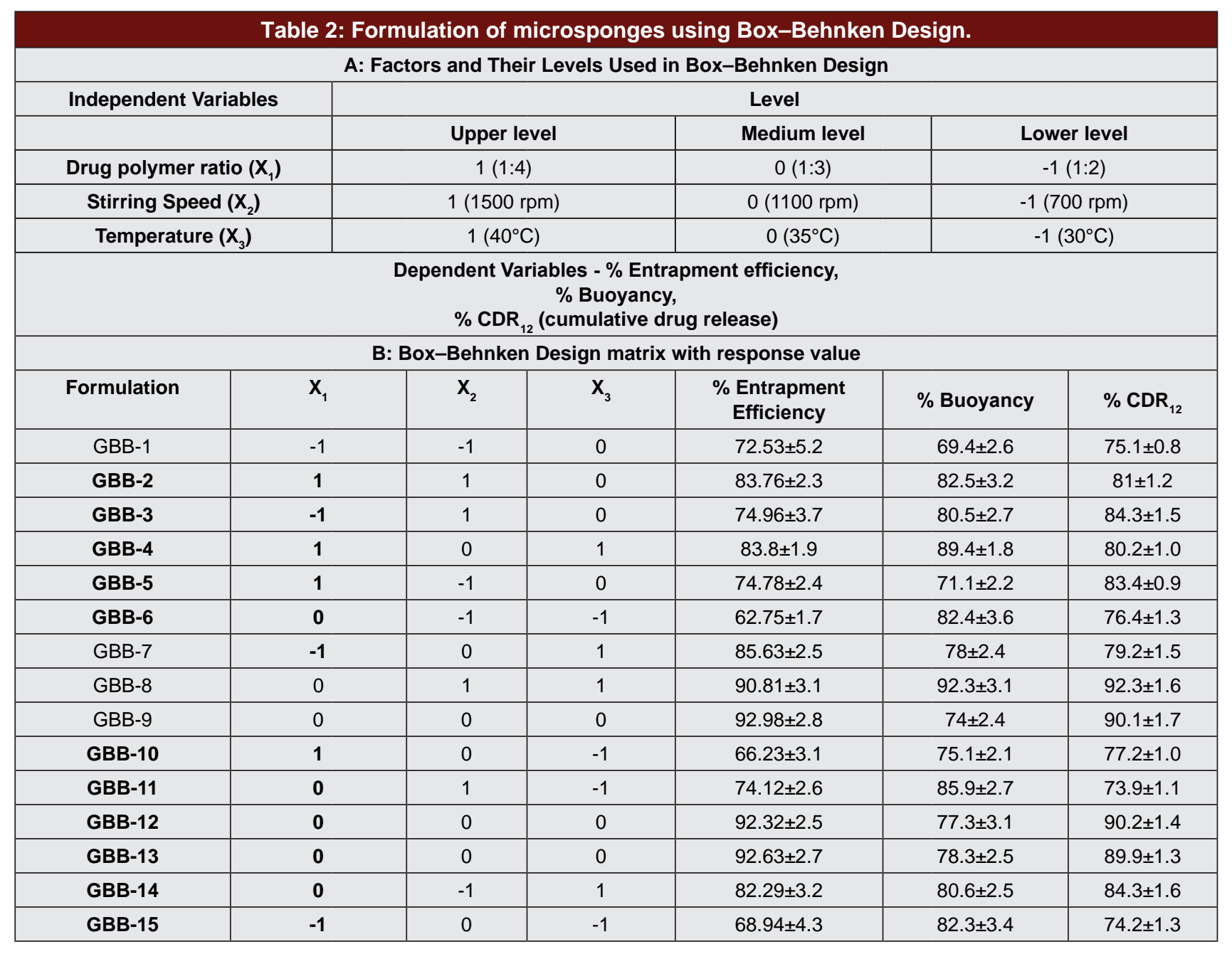

$$
\text { Bulk density }=\frac{\text { Weight of microsponges }}{\text { Initial volume }}
$$

\section{In vitro buoyancy}

The test was conducted by spreading $100 \mathrm{mg}$ microsponges over the surface of $100 \mathrm{ml}$ of $0.1 \mathrm{~N} \mathrm{HCl}$. The medium was agitated magnetically at a speed of 100 $\mathrm{rpm}$ at $37 \pm 0.5^{\circ} \mathrm{C}$ for $12 \mathrm{~h}$. After $12 \mathrm{hr}$, the floating and non-floating microsponges were filtered separately and dried. Both the portions of dried microsponges were weighed. The percent buoyancy was calculated by ratio of the floating microsponges to the total number of microsponges (floating and sinking). ${ }^{19}$

Where $W_{f}$ is weight of floating microsponges and $W_{n f}$ is weight of non-floating microsponges. All the determinations were made in triplicates.

\section{In vitro drug release}

The release of GLP from the microsponges was determined by filling microsponge's weight equivalent to
$10 \mathrm{mg}$ in an empty capsule shell and then the dissolution was carried out in USP dissolution apparatus II. The dissolution medium used was $500 \mathrm{ml}$ of $0.1 \mathrm{~N}$ $\mathrm{HCl}$, which was maintained at $37 \pm 0.5^{\circ} \mathrm{C}$ at $75 \mathrm{rpm}$. A sample of $5 \mathrm{ml}$ was withdrawn at every hour for $12 \mathrm{hr}$ and replaced with an equal volume of fresh medium to maintain the sink condition. Samples were filtered through membrane filter $(0.45 \mu \mathrm{m})$ and assayed using spectrophotometer at $276 \mathrm{~nm} .^{20}$ The release of the prepared gastroretentive formulations was compared with the sustained release marketed formulation (GLYTOP$\mathrm{SR}$ ) of the drug. All the dissolution studies were done in triplicates.

\section{Statistical analysis}

Design-Expert ${ }^{\circledR}$ software (trial version 9.0.6, Stat-Ease) was used to check the effect of independent variables on the responses, considered as dependent variables. Polynomial equations were generated for the dependent variables entrapment efficiency, buoyancy and CDR $12 \mathrm{~h} \%$. The optimized formulation was selected 
on the basis of high entrapment efficiency, buoyancy, CDR $12 \mathrm{~h} \%$ and maximum desirability.

\section{Validation of experimental design}

To validate the experimental design, an extra check point formulation was prepared. The values for entrapment efficiency, buoyancy and CDR $12 \mathrm{~h} \%$ were predicted by their respective polynomial equations. The check point batch had the composition with the levels as X1: 0.1, X2: 0.71 and X3: 0.99. The experimental values were determined by evaluating the dependent variables. The predicted and experimental values of the responses were compared for statistical significance using t-test at 95\% confidence interval $(p<0.05)$.

\section{Characterization of Microsponges}

Surface morphology of optimized formulation was visualized by scanning electron microscope from Sophisticated Instrumentation Centre for Applied Research and Testing (SICART), Anand, Gujarat, India (Model ESEM EDAX XL-30 Philips, Netherlands). The samples were coated with gold under argon atmosphere using gold sputter module in a high vacuum evaporator and observed under various magnifications (100-1000X) with direct data capture of the image. ${ }^{21}$

The Differential scanning calorimetry (DSC) thermograms of Pure GLP, ethyl cellulose and their physical mixture and optimized GLP floating microsponges were recorded using Shimadzu DSC-60, to confirm the compatibility. The samples were sealed in aluminum pans heated in an atmosphere of nitrogen and thermograms were obtained by heating at a constant heating rate of $10^{\circ} \mathrm{C} / \mathrm{min}$ in the range of $0-300^{\circ} \mathrm{C}$. A nitrogen purge $(40 \mathrm{ml} / \mathrm{min})$ was maintained throughout the run. ${ }^{22,23}$

Powder X-ray diffraction (XRD) for pure drug and optimized GLP microsponges was performed, at SICART, (Model No. Xpert MPD, Philips, Holland), to investigate the effect of polymerization on crystallinity of the drug. Over the angle range $(2 \theta) 0^{\circ}-60^{\circ}$, the samples were analyzed. ${ }^{24}$

Residual solvents are the left over organic solvents in the final finished pharmaceutical product, when such solvents are used for the preparation of the formulation. The ICH guidelines "Q3C" for the residual solvents, has given the permitted daily exposure (PDE) and concentration limit in ppm for these solvents. ${ }^{25}$ In the present work, dichloromethane (DCM) was used in the preparation of GLP microsponges. Hence, the Gas Chromatographic technique was applied to determine the amount of DCM (limit is upto 600ppm) in the optimized GLP microsponges (GBB-8). Formulation GBB-8 was tested by a 7697A Headspace (Agilent; Santa Clara, CA, USA) gas chromatograph (GC) with a DB 624 column (30 m $\times 450 \mu \mathrm{m} \times 2.55 \mu \mathrm{m})$ and flame ionization detector. For this study, $10 \mathrm{mg}$ of optimized microsponges of GBB-8, were dissolved in $5 \mathrm{~mL}$ of Dimethyl sulfoxide (DMSO) and transferred to the GC system. For calculations, a standard solution of dichloromethane in DMSO (20 ppm) was also analyzed. ${ }^{26}$

\section{Radiographic study}

The in-vivo radiographic studies were conducted on healthy albino rabbits weighing 2.0 to $2.2 \mathrm{~kg}$. The protocol (BIP/IAEC/2015/05) for in vivo study was approved by the Institutional Animal Ethical Committee (IAEC) in accordance with guidance of committee for the purpose of control and supervision of experiments on animals (CPCSEA). Floating microsponges was prepared by incorporating the X-ray opaque material in the optimized formulation by replacing glipizide with barium sulphate. The amount of the X-ray opaque material in the optimized formula of microsponge was kept sufficient to ensure visibility by X-ray, but at the same time the amount of barium sulphate was low enough to enable the formulation to float. This formulations was given to albino rabbit for in vivo X-ray imaging study. During the study the rabbit was not allowed to eat, but water was available freely. ${ }^{27}$

\section{RESULTS AND DISCUSSION}

\section{Screening of critical factors using Plackett and Burman design}

An attempt was made to develop a gastroretentive floating microsponges of Glipizide by quasi-emulsion solvent diffusion method. Screening of the formulation and process related factors by trial and error technique is time consuming and can be inaccurate at times. Hence, Plackett-Burman design was employed as the screening technique to determine the most significant factors that affected the formulation of microsponges using DesignExpert ${ }^{\circledR}$ software. The factor screened by the design were temperature $\left(\mathrm{X}_{1}\right)$, polymer concentration $\left(\mathrm{X}_{2}\right)$, stirring speed $\left(\mathrm{X}_{3}\right)$, amount of plasticizer $\left(\mathrm{X}_{4}\right)$, amount of PVA $\left(\mathrm{X}_{5}\right)$, volume of internal phase solvent $\left(\mathrm{X}_{6}\right)$, stirring time $\left(\mathrm{X}_{7}\right)$. The effect of these independent variables was checked on dependent variables ( $\%$ yield and \%entrapment efficiency).

\section{Effect of the variables on yield of the product}

The Pareto chart of yield (Figure 1) and the final equation indicated that the most significant factors for yield are $\mathrm{X}_{2}$ (drug-polymer ratio) and $\mathrm{X}_{3}$ (stirring speed). The $\mathrm{R}^{2}$ 


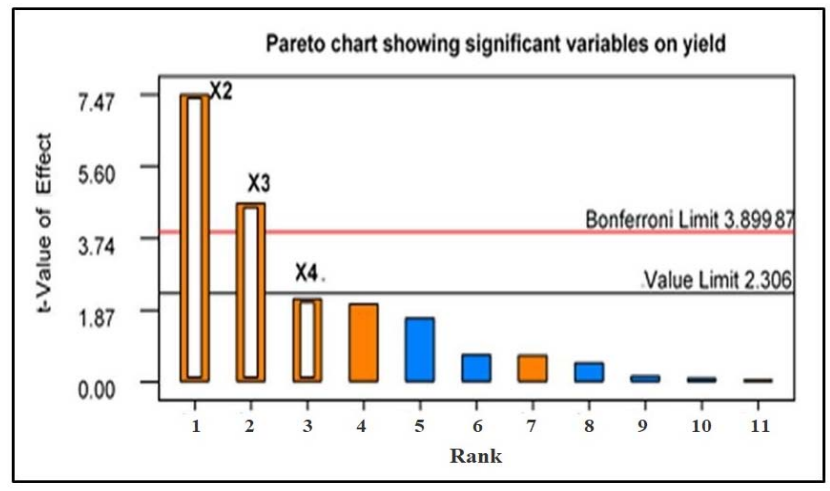

Figure 1: Plackett-Burman design for GLP microsponges Pareto Chart of yield.

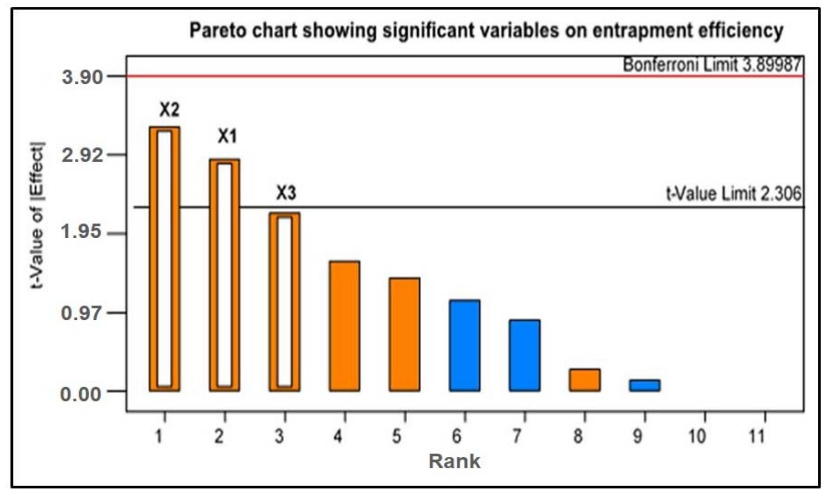

Figure 2: Plackett-Burman design for GLP microsponges Pareto Chart of Entrapment Efficiency.

value was found to be equal to 0.8485 , which means model is significant. The difference between adjusted and predicted $\mathrm{R}^{2}$ value was found to be less than 0.2 and also the $F$-value of 7.93 proved the significance of model.

$$
\text { Yield }(\%)=+73.83+9.71 \mathrm{X}_{2}+6.03 \mathrm{X}_{3}+2.80 \mathrm{X}_{4}
$$

The equation clearly shows that, as the drug polymer ratio increases (amount of polymer increases), the yield of micosponges increases. The outcomes were similar to the results obtaining earlier. ${ }^{28}$ The high level of ethyl cellulose makes the organic phase viscous which decreases the diffusion of organic phase to aqueous phase that prolongs polymer solidification thus increases the yields.

\section{Effect of the variables on entrapment efficiency}

The Pareto chart of entrapment efficiency (Figure 2) and the final equation clearly indicates that the most significant factors, are $\mathrm{X}_{1}$ (temperature) $\mathrm{X}_{2}$ (drug-polymer ratio) and $X_{3}$ (stirring speed), in terms of entrapment efficiency. The $R^{2}$ value was found to be equal to 0.9111 , which means good agreement between the dependent and independent variables. The difference between adjusted and predicted $R^{2}$ value was found to be less than 0.2 and also the F-value of 27.33 proved the significance of model.

Entrapment Efficiency $(\%)=+74.48+4.99 \mathrm{X}_{1}+5.68 \mathrm{X}_{2}+3.83 \mathrm{X}_{3}$

The combined effect of the selected variables on yield and entrapment efficiency indicated that concentration of polymer i.e. ethyl cellulose, stirring speed and temperature significantly affected the formulation of microsponges. They were considered to be the most critical factors in the formulation of microsponges with positive sign indicating their positive influence on dependent variables. Hence, these factors (temperature, drug-polymer ratio and stirring speed) were further evaluated by applying Box-Behnken design to get the optimized formulation of GLP microsponges.

\section{Optimization of GLP Microsponges: Box-Behnken Design}

The result (Table 3) shows that the percent yield for all the batches was in the range of $84.8 \%$ to $98.8 \%$. The particle size was found to be in the orbit of 96.30 to $243.87 \mu \mathrm{m}$, which comes in the standard range $(5-300 \mu \mathrm{m})$ of microsponges as mentioned by Nacht et al. ${ }^{29}$ General observation was that as the stirring speed increased the particle size decreased as earlier proved..$^{21}$ The polymer concentration had an optimistic effect on particle size, as the concentration of ethyl cellulose increased, the particle size also increased. This is because, as the concentration of polymer in the organic phase increases, the emulsification is hindered and big droplets of polymer are formed during the polymerization, leading to bigger particle size.

Bulk density was found to be in the range of 0.087 to $0.384 \mathrm{~g} / \mathrm{cc}$. The high concentration of polymer makes the solution thick, hence particle size increases. Whereas, high stirring speed makes the fine droplet of polymer which result in smaller particle size.

The drug release study was performed for all the prepared box-behnken design batches. The release profile of all batches was compared with standard (STD) marketed sustained release formulation of GLP (Figure 3). A general observation was that the batches prepared with medium concentration of polymer gave better release pattern. Only these batches could pass the criteria of model independent analysis of dissolution.

The batches prepared with the high concentration of ethyl cellulose showed delayed release of the drug, 


\begin{tabular}{|c|c|c|c|c|c|c|}
\hline \multicolumn{7}{|c|}{ Table 3: Result table of GLP Microsponges prepared by applying Box-Behnken Design. } \\
\hline Formulation & \% Yield & $\begin{array}{c}\text { Particle size } \\
(\boldsymbol{\mu} \mathbf{m})\end{array}$ & $\begin{array}{c}\text { Bulk density } \\
(\mathbf{g} / \mathbf{c c})\end{array}$ & $\begin{array}{c}\text { \% Entrapment } \\
\text { Efficiency }\end{array}$ & \% Buoyancy & \% CDR \\
\hline GBB-1 & 96.4 & $157.43 \pm 14.6$ & 0.222 & $72.53 \pm 5.2$ & $69.4 \pm 2.6$ & $75.1 \pm 0.8$ \\
\hline GBB-2 & 98.4 & $172.4 \pm 11.4$ & 0.106 & $83.76 \pm 2.3$ & $82.5 \pm 3.2$ & $81 \pm 1.2$ \\
\hline GBB-3 & 97.6 & $114.18 \pm 10.5$ & 0.113 & $74.96 \pm 3.7$ & $80.5 \pm 2.7$ & $84.3 \pm 1.5$ \\
\hline GBB-4 & 98.88 & $143.25 \pm 15.7$ & 0.101 & $83.8 \pm 1.9$ & $89.4 \pm 1.8$ & $80.2 \pm 1.0$ \\
\hline GBB-5 & 84.8 & $153.97 \pm 16.9$ & 0.384 & $74.78 \pm 2.4$ & $71.1 \pm 2.2$ & $83.4 \pm 0.9$ \\
\hline GBB-6 & 86 & $104.37 \pm 13.6$ & 0.107 & $62.75 \pm 1.7$ & $82.4 \pm 3.6$ & $76.4 \pm 1.3$ \\
\hline GBB-7 & 85.3 & $127.54 \pm 10.4$ & 0.202 & $85.63 \pm 2.5$ & $78 \pm 2.4$ & $79.2 \pm 1.5$ \\
\hline GBB-8 & 96.3 & $243.87 \pm 9.3$ & 0.087 & $90.81 \pm 1.21$ & $92.3 \pm 2.25$ & $92.3 \pm 0.9$ \\
\hline GBB-9 & 93.7 & $138.74 \pm 18.5$ & 0.219 & $92.98 \pm 2.8$ & $74 \pm 2.4$ & $90.1 \pm 1.7$ \\
\hline GBB-10 & 70.96 & $96.3 \pm 12.5$ & 0.217 & $66.23 \pm 3.1$ & $75.1 \pm 2.1$ & $77.2 \pm 1.0$ \\
\hline GBB-11 & 93.2 & $195.67 \pm 12.7$ & 0.122 & $74.12 \pm 2.6$ & $85.9 \pm 2.7$ & $73.9 \pm 1.1$ \\
\hline GBB-12 & 92.5 & $129.74 \pm 10.5$ & 0.210 & $92.32 \pm 2.5$ & $77.3 \pm 3.1$ & $90.2 \pm 1.4$ \\
\hline GBB-13 & 90.2 & $133.74 \pm 16.2$ & 0.225 & $92.63 \pm 2.7$ & $78.3 \pm 2.5$ & $89.9 \pm 1.3$ \\
\hline GBB-14 & 91.4 & $119.66 \pm 17.6$ & 0.114 & $82.29 \pm 3.2$ & $80.6 \pm 2.5$ & $84.3 \pm 1.6$ \\
\hline GBB-15 & 92.6 & $125.9 \pm 13.2$ & 0.11 & $68.94 \pm 4.3$ & $82.3 \pm 3.4$ & $74.2 \pm 1.3$ \\
\hline
\end{tabular}

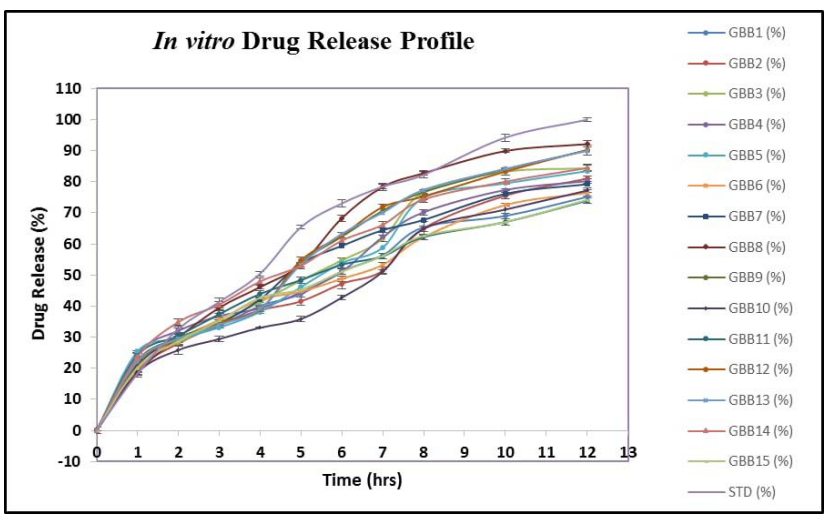

Figure 3: In vitro drug release profile of microsponge batches prepared using Box-Behnken design.

which is because of the fact that more polymer concentration increases path length, which the drug molecule has to cross. Earlier finding suggest that the reason for getting delayed release with high concentration of polymer is related to decreases the amount of drug close to surface of microsponges exposed to the dissolution medium. This leads to lowering in the rate of drug release from the microsponges. The release pattern of formulation GBB-8 had the maximum similarity factor $\left(f_{2}\right)$ value with marketed formulation.

\section{Statistical Analysis}

The result of all the dependent variables is given in Table 4. For Box-Behnken analyses, the regression equation describes the effects of the variables on the responses in terms of linear, interactive and quadratic.

\begin{tabular}{|c|c|c|c|c|c|}
\hline Source & $\begin{array}{l}\text { Sum of } \\
\text { Squares }\end{array}$ & $\begin{array}{c}\text { Degree } \\
\text { of } \\
\text { freedom }\end{array}$ & $\begin{array}{l}\text { Mean } \\
\text { Square }\end{array}$ & $\begin{array}{c}F \\
\text { Value }\end{array}$ & $P$-value \\
\hline \multicolumn{6}{|c|}{ Buoyancy (\%) } \\
\hline Model & 537.61 & 9 & 59.73 & 14.59 & 0.0044 \\
\hline Residual & 20.46 & 5 & 4.09 & & \\
\hline $\begin{array}{c}\text { Corrected } \\
\text { Total }\end{array}$ & 558.08 & 14 & & & \\
\hline \multicolumn{6}{|c|}{ Entrapment Efficiency (\%) } \\
\hline Model & 1374.46 & 9 & 152.72 & 19.05 & 0.0023 \\
\hline Residual & 40.07 & 5 & 8.01 & & \\
\hline $\begin{array}{c}\text { Corrected } \\
\text { Total }\end{array}$ & 1414.53 & 14 & & & \\
\hline \multicolumn{6}{|c|}{$\mathrm{CDR}_{12}(\%)$} \\
\hline Model & 511.99 & 9 & 56.89 & 6.73 & 0.0246 \\
\hline Residual & 42.24 & 5 & 8.45 & & \\
\hline $\begin{array}{c}\text { Corrected } \\
\text { Total }\end{array}$ & 554.24 & 14 & & & \\
\hline
\end{tabular}

The polynomial equation generated by Box-Behnken design using Design expert software is as follows:

$$
\begin{aligned}
& Y=b_{0}+b_{1} X_{1}+b_{2} X_{2}+b_{3} X_{3}+b_{12} X_{1} X_{2}+ \\
& b_{13} X_{1} X_{3}+b_{23} X_{2} X_{3}+b_{11} X_{1}^{2}+b_{22} X_{2}^{2}+b_{33} X_{3}^{2}
\end{aligned}
$$

Where, $\mathrm{Y}$ is the dependent variable, $\mathrm{b}_{0}$ is the intercept and $b_{1}$ to $b_{33}$ are regression coefficient. The master 

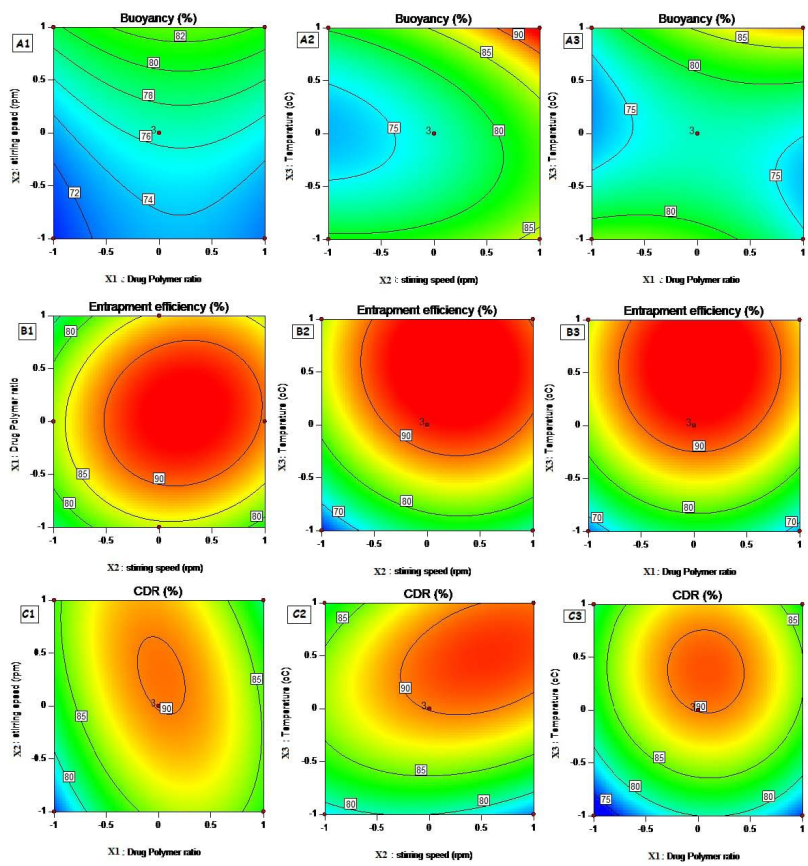

X1: Ding Patmer nate

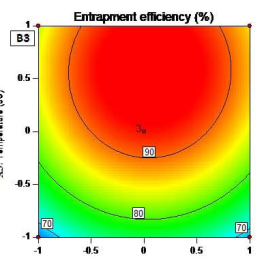

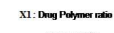
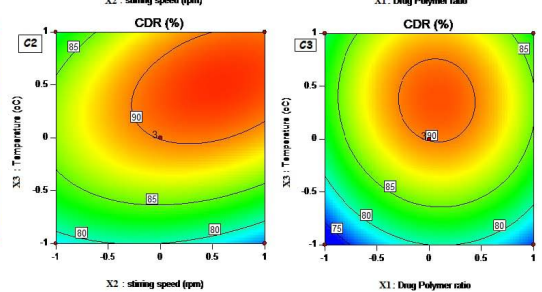

Figure 4: Contour plot of buoyancy (A), entrapment efficiency (B) and CDR12 (C).

effects $\left(X_{1}, X_{2}\right.$ and $\left.X_{3}\right)$ represent the average result of changing one element at a time from its low to high value. $X_{1} X_{2}, X_{1} X_{3}$ and $X_{2} X_{3}$ represents the interaction terms and $X_{1}^{2}, X_{2}^{2}$ and $X_{3}^{2}$ represents quadratic effect. Using analysis of variance (ANOVA), the significance $(p<0.05)$ of the ratio of mean square variation due to the regression coefficient and the residual error were tested. The summary of ANOVA table for response parameters is given in Table 4. The Quadratic model was found to be significant for all the responses. The $p$-value was found to be significant, which indicates that the model was significant. The high values of correlation coefficients for buoyancy $\left(\mathrm{R}^{2}=0.9633\right)$, Entrapment efficiency $(R 2=0.9717)$ and $\mathrm{CDR}_{12}(\mathrm{R} 2=0.9238)$ indicated a good fit (i.e, good agreement between the dependent and independent variables).

\section{Effect of independent variables on Buoyancy}

The percent of entrapment efficiency was found to be in the range of 69.4 to $92.3 \%$. P value was found to be 0.0044 implies the model is significant. $R^{2}$ was found to be equal to 0.9633 . The established second-degree polynomial equation for entrapment efficiency was as follow:

$$
\begin{array}{r}
\operatorname{Buoyancy}(\%)=+76.53+0.99 \mathrm{X}_{1}+4.71 \mathrm{X}_{2}+1.82 \mathrm{X}_{3}+0.075 \mathrm{X}_{1} \mathrm{X}_{2}+ \\
4.65 \mathrm{X}_{1} \mathrm{X}_{3}+2.05 \mathrm{X}_{2} \mathrm{X}_{3}-2.38 \mathrm{X}_{1}^{2}+1.72 \mathrm{X}_{2}^{2}+7.05 \mathrm{X}_{3}^{2}
\end{array}
$$

The equation indicates that all the independent variables have a positive influence on the buoyancy of the micro- sponges. The most important element was found to be stirring speed $\left(\mathrm{X}_{2}\right)$, as the speed of stirring increased the buoyancy also increased. There was a significant positive interaction effect found with drug polymer ratio $\left(\mathrm{X}_{1}\right)$ and temperature $\left(\mathrm{X}_{3}\right)$. A quadratic effect of temperature was also noted and these effects were further illustrated in contour plots (Figure 4).

The effects of $\mathrm{X}_{1}$ and $\mathrm{X}_{2}$ with their interaction on buoyancy at a specified level of $\mathrm{X}_{3}(0)$ are shown in contour plot, Figure 4 (A1). The plots were found to be linear at $72 \%$ buoyancy, but above this value, the plots were found to be nonlinear indicating a nonlinear relationship between $\mathrm{X}_{1}$ and $\mathrm{X}_{2}$ with maximum nonlinearity found with $74 \%$ of buoyancy. It was learned from the contour plot that the maximum buoyancy $82 \%$ was noted at the highest value of $\mathrm{X}_{2}$ and the value of $\mathrm{X}_{1}$ maintained between the ranges 0 to 0.5 . The contour plot of $X_{2}$ and $\mathrm{X}_{3}$ showed that highest non linearity was observed at buoyancy below $85 \%$ and for obtaining the maximum buoyancy the values of $X_{2}$ and $X_{3}$ should be near 1 [Figure 4 (A2)]. Contour plot of buoyancy drawn at 0 values of $\mathrm{X}_{2}$ showed that $85 \%$ of buoyancy is achieved with highest value of both the independent variables [Figure 4 (A3)]. Overall, for buoyancy, the most significant factor was stirring speed followed by temperature of the medium. This indicated that as the stirring speed increases, the porosity of microsponge increases and formulation becomes more buoyant.

\section{Effect of independent variables on Entrapment Efficiency}

The percent of entrapment efficiency was found to be in the range of 62.75 to $92.98 \%$. $P$ value was found to be 0.0023 implies the model is significant. $R 2$ was found to be equal to 0.9715 . The established seconddegree polynomial equation for entrapment efficiency was as follow:

$$
\begin{aligned}
& \text { Entrapment Efficiency }(\%)= \\
& +92.64+0.81 \mathrm{X}_{1}+3.91 \mathrm{X}_{2}+ \\
& 8.81 \mathrm{X}_{3}+1.64 \mathrm{X}_{1} \mathrm{X}_{2}+0.22 \mathrm{X}_{1} \mathrm{X}_{3}- \\
& 0.71 \mathrm{X}_{2} \mathrm{X}_{3}-8.74 \mathrm{X}_{1}^{2}-7.40 \mathrm{X}_{2}^{2}-7.75 \mathrm{X}_{3}^{2}
\end{aligned}
$$

The equation indicates that all the independent variables have a positive influence on the entrapment efficiency of the microsponges. The significant antagonistic quadratic effect of the independent factors was observed on entrapment efficiency, which was further shown by contour plots. A contour plot is a graphical technique for representing a 3-dimensional surface by plotting constant $\mathrm{z}$ slices, called contours, on a 2-dimensional format. This implies that the result of two variables can be 
pulled out by holding open the third variable constant. Here, the plots suggested that $90 \%$ of entrapment efficiency can be achieved with $\mathrm{X}_{1}$ in the range of -0.6 to 0.75 and $\mathrm{X}_{2}$ in the range of -0.5 to 0.9 at a fixed level of $\mathrm{X}_{3}(0)$ are shown in contour plot, Figure 4 (B1). The contour plot of $X_{2}$ and $X_{3}$ with $X_{1}$ constant, showed that highest entrapment efficiency can be obtained when $\mathrm{X}_{3}$ is kept in the range of -0.2 to 1 and $\mathrm{X}_{2}$ in the range of -0.6 to 1 [Figure 4 (B2)]. Contour plot of entrapment efficiency, drawn at 0 value of $X_{2}$ showed that the value of entrapment efficiency was more in the range of $\mathrm{X}_{3}$ as -0.2 to 1 and $\mathrm{X}_{1}$ as -0.7 to 0.7 [Figure 2 (B3)]. As the quadratic effect is significant, it means that optimal levels of $\mathrm{X}$ are not in the extremes of the experimental region but inside it. However, as per the linear effect for entrapment efficiency, the most significant factor was the temperature of the medium. As the temperature increased fast solidification of the dispersed phase helped in faster solidification of polymer which prevents drug diffusion across the phase boundary and hence more entrapment of the drug.

\section{Cumulative drug release (CDR $12 \mathrm{hr}$ )}

Microsponges offer the controlled release of the drug as proven during earlier studies. ${ }^{30}$ The percent $\mathrm{CDR}_{12}$ was found to be in the range of 73.9 to $92.3 \%$. P value was found to be 0.0246 implies the model is significant. R2 was found to be equal to 0.9838 . This model can be used to navigate the design space.

The established second-degree polynomial equation for entrapment efficiency was as follow:

$$
\begin{aligned}
& \mathrm{CDR}_{12}(\%)=+90.07+1.12 \mathrm{X}_{1}+1.54 \mathrm{X}_{2}+4.29 \mathrm{X}_{3}- \\
& 2.90 \mathrm{X}_{1} \mathrm{X}_{2}-0.50 \mathrm{X}_{1} \mathrm{X}_{2}+2.63 \mathrm{X}_{2} \mathrm{X}_{3}- \\
& 6.57 \mathrm{X}_{1}^{2}-2.55 \mathrm{X}_{2}^{2}-5.80 \mathrm{X}_{3}^{2}
\end{aligned}
$$

The equation indicates that all the independent variables have a positive influence on the $\mathrm{CDR}_{12}$ of the microsponges. The significant antagonistic quadratic effect of the independent factors was observed on $\mathrm{CDR}_{12}$, which was further shown by contour plots. A contour plot suggested plots suggested that $90 \%$ of $\mathrm{CDR}_{12}$ can be achieved with $\mathrm{X}_{1}$ in the range of -0.3 to 0.3 and $X_{2}$ in the range of 0 to 0.5 at a fixed level of $X_{3}$ (0) are shown in contour plot, Figure 4 (C1). The contour plot of $\mathrm{X}_{2}$ and $\mathrm{X}_{3}$ with $\mathrm{X}_{1}$ constant, showed that highest $\mathrm{CDR}_{12}$ can be obtained when $\mathrm{X}_{3}$ is kept in the range of 0 to 1 and $X_{2}$ in the range of -0.7 to 1 [Figure 4 (C2)]. Contour plot of entrapment efficiency, drawn at 0 value of $X_{2}$ showed that the value of $C_{12}$ was more in the range of $\mathrm{X}_{3}$ as 0 to 0.8 and $\mathrm{X}_{1}$ as -0.3 to 0.4
[Figure 2 (C3)]. Overall, for $\mathrm{CDR}_{12}$, interaction within the independent variable was observed.

\section{Validation of Experimental design}

In order to validate the experimental design, the check point batch was prepared and evaluated for all the dependent responses. The composition and results for the check point batch is given in Table 4. The predictive and actual values for entrapment efficiency was found to be $91.7606 \%$ and $91.67 \%$ respectively. The predictive and actual values for buoyancy was found to be $92.3 \%$ and $93.92 \%$ respectively. The predictive and actual values for $\mathrm{CDR}_{12}$ was found to be $90.0582 \%$ and $90.9 \%$ respectively. The relative error (\%) between the predicted and actual values for each response was calculated and the values were found to be within 5\%. The experimental values were in agreement with the predicted values confirming the validity of the model.

\section{Selection of Optimized Batch}

To optimize all the responses with different targets, a numerical optimization technique by the desirability function and a graphical optimization technique by the overlay plot was used. The optimized formulation was obtained by applying constraints on dependent variable responses and independent variables. The constraints for all the dependent variables were set at $80 \%$ to $100 \%$. The recommended concentrations of the independent variables were calculated by the Design Expert software from the overlay plots obtained which has the highest desirability near to 1.0. Using design expert software three overlay plots were obtained indicating the area of optimal process variables as applied. Figure 5 (A), represents an overlay plot obtained with variable $\mathrm{X}_{1}$ (drug polymer ratio) and $\mathrm{X}_{2}$ (stirring speed) by keeping $\mathrm{X}_{3}$ (temperature) constant as 0.993937 . Overlay plot, Figure 5 (B), was obtained with $X_{1}$ and $X_{3}$ with $X_{2}$ constant as 0.7948 and Figure $5(\mathrm{C})$, overlay was obtained with $\mathrm{X}_{2}$ and $\mathrm{X}_{3}$ after fixing the value of $\mathrm{X}_{1}$ as 0.09930. After applying the desirability criteria and looking into overlay plots, formulation GBB-8 with medium drug- polymer ratio and maximum level of

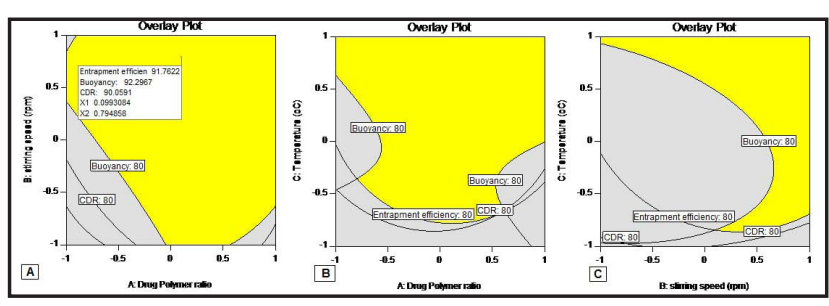

Figure 5: Overlay Plot of GLP floating Microsponges by Box-Behnken design. 
the other two variables was found to be optimum with desirability near 1 . The formulation gave entrapment efficiency as $90.81 \%$, buoyancy as $92.3 \%$ and CDR $12 \mathrm{hr}$ as $92.3 \%$.

This clearly indicates that glipizide microsponges are best formulated with 1:3 drug polymer ratio, $40^{\circ} \mathrm{C}$ temperature and $1500 \mathrm{rpm}$ stirring speed. Formulation GBB-8 was considered to be the optimized formulation and it was used for physicochemical characterization of floating microsponges of GLP and also for the in vivo studies.

\section{Physicochemical characterization of Glipizide Microsponges}

Powder X-ray diffraction (XRD) was performed for optimized microsponge formulation of Glipizide to investigate the effect of polymerization on crystallinity of the drug. The XRD scan of glipizide microsponges was compared with the XRD scan of pure drug (Figure 6). The distinct peaks can be observed in the XRD of pure glipizide which shows the crystalline behavior of the drug. The disappearance of most of the characteristic peaks of the drug in the formulation indicates that most of the drug has been converted to the amorphous form and drug is dispersed at a molecular level in the polymeric matrix. The interpretation of the results achieved was matching with the XRD analysis obtained by Deshmukh et al. ${ }^{24}$

DSC provides the information about the crystalline and amorphous form of drug and possible interaction during the polymerization and formulation of microsponges. The thermogram of pure Glipizide exhibited a sharp endothermic peak at $209.62^{\circ} \mathrm{C}$ corresponding to its melting point $\left(208-209^{\circ} \mathrm{C}\right)$, representing its crystalline nature. The thermogram of pure ethyl cellulose gives no sharp peak. The thermogram of physical mixture of polymer and drug and formulation GBB-8, gave

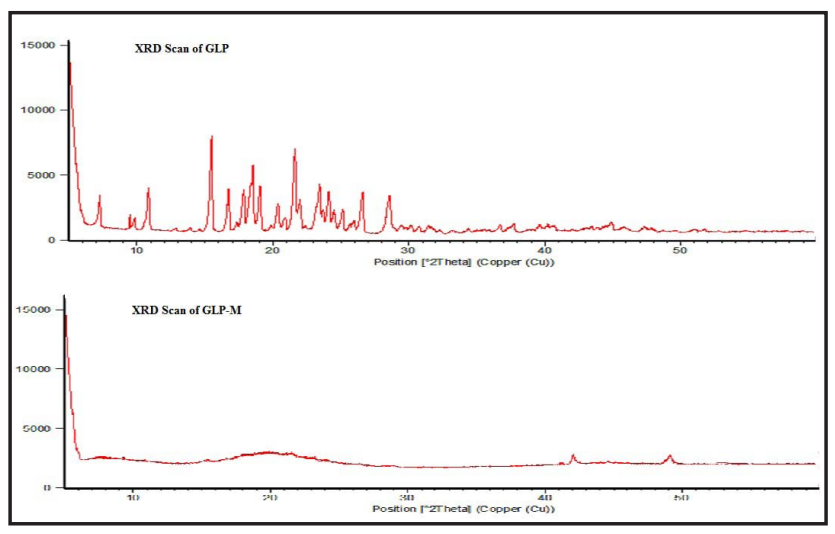

Figure 6: XRD scan of Glipizide and batch GBB-8.

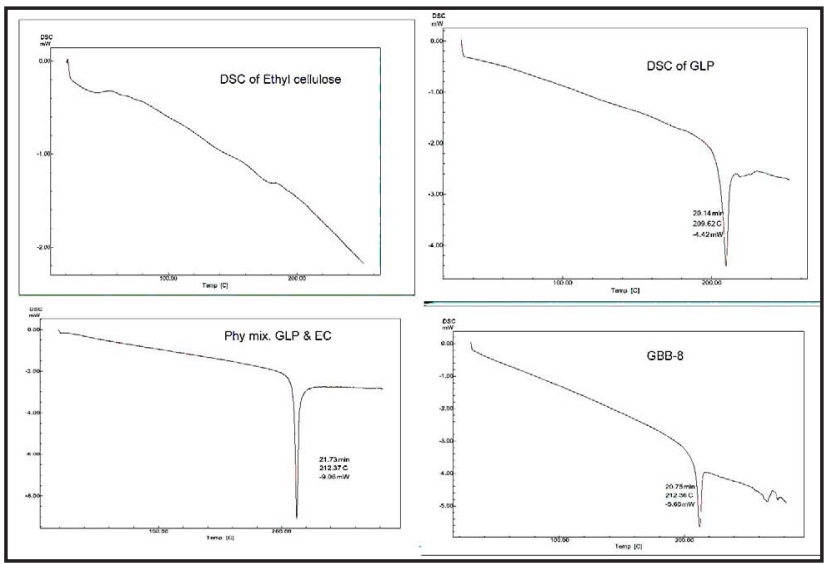

Figure 7: DSC scan of Glipizide, Ethyl cellulose, Physical mixture of drug and polymer, optimized formulation.

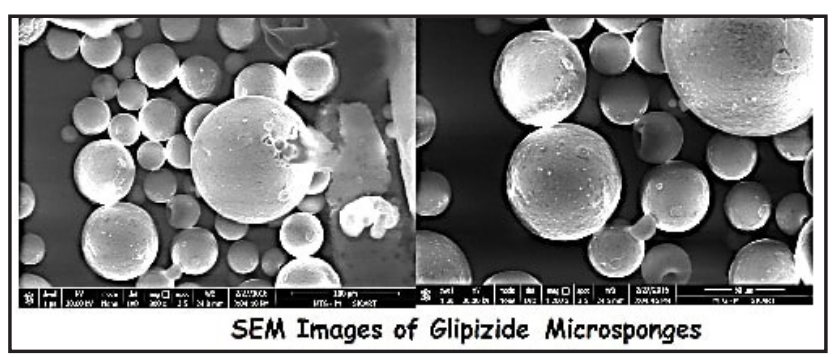

Figure 8: SEM images of Glipizide microsponges.

the peaks at $212.37^{\circ} \mathrm{C}$ and $212.36^{\circ} \mathrm{C}$, respectively, with decrease in the intensity of peak (Figure 7). There was slight increase in the peak temperature in the both, which may be attributed to the presence of ethyl cellulose and the dispersion of drug in polymeric matrix.

SEM images of optimized batch of GLP microsponges (GBB-8) were taken to get the topographical information about the formulation. The results revealed that the formulated GLP microsponges are smooth surfaced spherical and highly porous in nature (Figure 8). Furthermore, no drug crystals were observed over the surface of formulation.

The organic solvents used for the formulation of GLP microsponges were ethanol and dichloromethane. Ethanol is considered as class 3 residual solvent which are regarded as less toxic as they have no human health hazard at levels normally accepted in pharmaceuticals prescribed by the ICH guidelines "Q3C" for the residual solvents. ${ }^{31}$ Hence, it is not essential to find the residual amount of ethanol in the finished product. But, DCM belongs to class 1 residual solvents and its amount in the finished formulation should be within limit (up to $600 \mathrm{ppm}$ ). The gas chromatogram of standard DCM solution in DMSO and GBB-8 dissolved in DMSO 


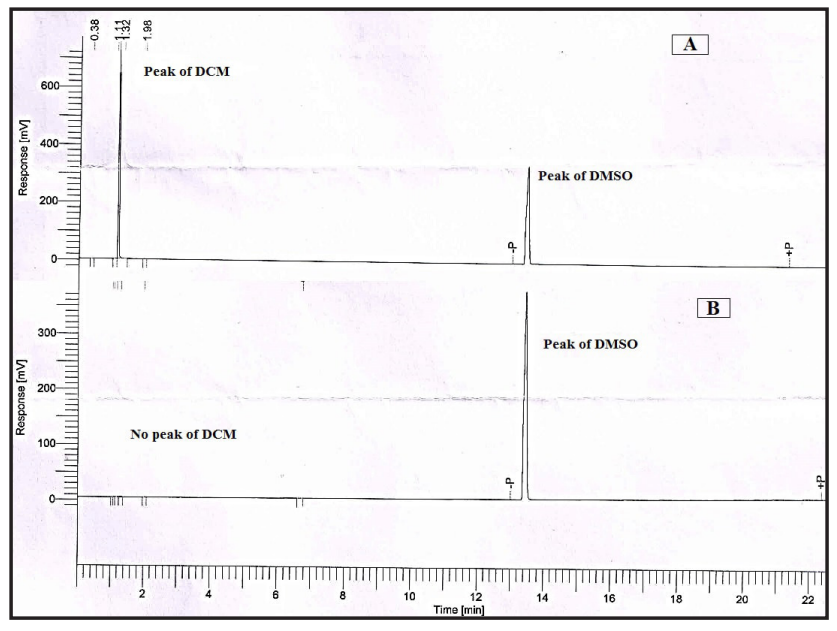

Figure 9: GC scan for DCM residual solvent in GLP floating microsponges A) Scan of standard DCM solution; B) Scan of GBB-8 formulation, solution prepared in DMSO.

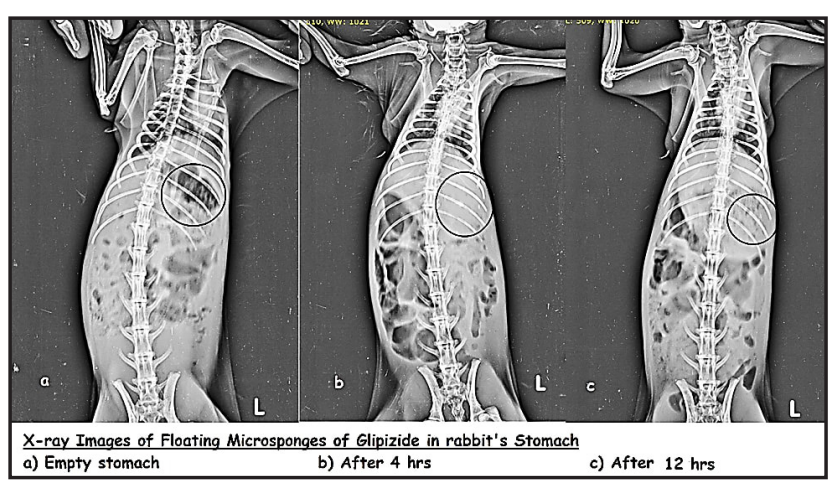

Figure 10: X-ray images of floating microsponges.

(Figure 9). The retention time of dichloromethane was $1.181 \mathrm{~min}$ as observed in standard preparation of DCM. The peak of DMSO was observed at $13.4 \mathrm{~min}$ in both standard and sample preparation. But, no dichloromethane peak at 1.181 min was observed in sample chromatogram. Dichloromethane residue was within the limits, in the GLP loaded microsponges. Hence, the prepared formulation GBB- 8 is considered to be safe for human use.

\section{Radiographic Study}

To determine the retention time of the optimized floating microsponges of GLP, in stomach, radiographic studies were conducted. The floating barium sulfate loaded microsponges were given to rabbits. The X-ray photomicrographs were taken immediately at 0,4 and $12 \mathrm{~h}$ and were recorded (Figure 10). The in vivo X-ray imaging study clearly indicated that the optimized formulation of microsponges remained afloat in gastric fluid up to $12 \mathrm{~h}$ in the stomach of rabbit. Hence, it is believed that the developed gastroretentive microsponges of GLP will remain buoyant in the stomach of human being as well.

\section{CONCLUSION}

It could be concluded that application of experimental design is helpful tool for the development of floating microsponges of glipizide.

During the preliminary studies, it was found that there are various factors that affected the formulation. Screening of these formulation and process related factors, by trial and error technique, is time consuming and can be inaccurate at times. Hence, Plackett-Burman design was employed as the screening technique to determine the most significant factors that affected the formulation of microsponges using Design-Expert ${ }^{\circledR}$ software. Pareto charts revealed that concentration of polymer i.e. ethyl cellulose, stirring speed and temperature were the most critical factors in the formulation of microsponges. Hence, these factors were further used for the final optimization of glipizide floating microsponges by applying Box-Behnken design. The dependent responses including entrapment efficiency, buoyancy and CDR12h \% were evaluated and results were statistically analyzed by Design expert software. The desirability function and overlay plot indicated GBB-8 (with $\mathrm{X}_{1}$ at 0 -level and $\mathrm{X}_{2}, \mathrm{X}_{3}$ at 1-level), as optimized formulation. The physicochemical characterization of optimized formulation showed no interaction between the drug and polymer and the complete dispersion of the drug in polymeric matrix and also the porous, spherical nature of the formulation. The radiographic studies performed on the albino rabbits proved the gastroretention of formulation in test animal for $12 \mathrm{hr}$. Hence, it is believed that the developed gastroretentive microsponges of GLP will remain buoyant in the stomach of human being as well. The prepared formulation can offer the sustained release of the drug at its site of absorption which may provide the better control of the diabetes due to less fluctuation in plasma drug concentration.

\section{ACKNOWLEDGEMENT}

The authors express their gratitude to Babaria Institute of Pharmacy, BITS Edu Campus for providing necessary support in due course of the work.

\section{CONFLICT OF INTEREST}

The authors declare that there are no conflicts of interest. 


\section{ABBREVIATIONS}

GLP: Glipizide; PB: Plackett-Burman; PVA: polyvinyl alcohol; SEM: scanning electron microscope; DCM: dichloromethane; TEC: triethylcitrate; DMSO: Dimethyl sulfoxide; DSC: Differential scanning calorimetry; XRD: X-ray diffraction; CDR: cumulative drug release; GC: Gas Chromatography; ANOVA: analysis of variance; ICH: International Council for Harmonization.

\section{REFERENCES}

1. Arya P, Pathak K. Assessing the viability of microsponges as gastro retentive drug delivery system of curcumin: Optimization and pharmacokinetics. Int J Pharm. 2014;460(1-2):1-12.

2. Pandya N, Pandya M, Bhaskar VH. Preparation and in vitro characterization of porous carrier-based glipizide floating microspheres for gastric delivery. J Young Pharm. 2011;3(2):97-104.

3. Foster RH, Plosker GL. Glipizide: A Review of the Pharmacoeconomic Implications of the Extended-Release Formulation in Type 2 Diabetes Mellitus. Pharmacoeconomics. 2000;18(3):289-306.

4. Chowdary KR, Rao NK, Malathi K. Ethyl cellulose microspheres of glipizide: Characterization, in vitro and in vivo evaluation. Indian J Pharm Sci. 2004;66(4):412-6.

5. Li VH, Robinson JR, Lee VH. Influence of drug properties and routes of drug administration on the design of sustained and controlled release systems. Controlled Drug Delivery. 1987;29(2):4-94.

6. Patel JK, Patel RP, Amin AF, et al. Formulation and evaluation of mucoadhesive glipizide microspheres. AAPS Pharm Sci Tech. 2005;6(1):E49-55.

7. Plackett RL, Burman JP. The design of optimum multifactorial experiments. Biometrika. 1946;33(4):305-25.

8. Bolzinger MA, Bordes C, Gauvrit JY, et al. Improvement of a bovine serum albumin microencapsulation process by screening design. Int $\mathrm{J}$ Pharm. 2007;344(1-2):16-25.

9. El-Malah Y, Nazzal S. Hydrophilic matrices: Application of Placket-Burman screening design to model the effect of POLYOX-carbopol blends on drug release. Int J Pharm. 2006;309(1):163-70.

10. Box GE, Behnken DW. Some new three level designs for the study of quantitative variables. Technometrics. 1960;2(4):455-75.

11. Solanki AB, Parikh JR, Parikh RH. Formulation and optimization of piroxicam proniosomes by 3-factor, 3-level Box-Behnken design. AAPS Pharm Sci Tech. 2007;8(4):43-9.

12. Çomoğlu T, Gönül N, Baykara T. Preparation and in vitro evaluation of modified release ketoprofen microsponges. II Farmaco. 2003;58(2):101-6.

13. Vyas S, Trivedi P, Chaturvedi SC. Dextran-etodolac conjugates: synthesis, in vitro and in vivo evaluation. Acta Pol Pharm Drug Res. 2009;66(2):201-6.
14. Re MI, Biscans B. Preparation of microspheres of ketoprofen with acrylic polymers by a quasi emulsion solvent diffusion method. Powder Technol. 1999;101(2):120-33.

15. Crcarevska MS, Dimitrovska A, Sibinovska N, et al. Implementation of quality by design principles in the development of microsponges as drug delivery carriers: Identification and optimization of critical factors using multivariate statistical analyses and design of experiments studies. Int $\mathrm{J}$ Pharm. 2015;489(1-2):58-72.

16. Sastry SV, Khan MA. Aqueous based polymeric dispersion: Plackett-Burman design for screening of formulation variables of atenolol gastrointestinal therapeutic system. Pharm Acta Helv. 1998;73(2):105-12.

17. Orlu M, Cevher E, Araman A. Design and evaluation of colon specific drug delivery system containing flurbiprofen microsponges. Int $\mathrm{J}$ Pharm. 2006;318(1):103-17.

18. Kilicarslan M, Baykara T. The effect of the drug/polymer ratio on the properties of verapamil hydrochloride loaded microspheres. Int J Pharm. 2003;252(12):99-109.

19. Pandit V, Pai RS, Yadav V, et al. Pharmacokinetic and pharmacodynamic evaluation of floating microspheres of metformin hydrochloride. Drug Dev Ind Pharm. 2013;39(1):117-27.

20. Phutane P, Lotlikar V, Ghule A, et al. In vitro evaluation of novel sustained release microspheres of glipizide prepared by the emulsion solvent diffusionevaporation method. J Young Pharm. 2010;2(1):35-41.

21. Nokhodchi A, Jelvehgari M, Siahi MR, et al. Factors affecting the morphology of benzoyl peroxide microsponges. Micron. 2007;38(8):834-40.

22. Jones DS, Pearce KJ. An investigation of the effects of some process variables on the microencapsulation of propranolol hydrochloride by the solvent evaporation method. Int J Pharm. 1995;118(2):199-205.

23. Kawashima $\mathrm{Y}$, Niwa $\mathrm{T}$, Takeuchi $\mathrm{H}$, et al. Characterization of polymorphs of tranilast anhydrate and tranilast monohydrate when crystallized by two solvent change spherical crystallization techniques. J Pharm Sci. 1991;80(5):472-8.

24. Deshmukh RK, Naik JB. Aceclofenac microspheres: Quality by design approach. Mater Sci Eng C. 2014;36:320-8.

25. ICH Topic Q3C (R4) Impurities: Guideline for Residual Solvents. European Medicines Agency. 2010;3-22.

26. Yerlikaya F, Ozgen A, Vural I, et al. Development and Evaluation of Paclitaxel Nanoparticles Using a Quality-by-Design Approach. J Pharm Sci. 2013;102(10):3748-61.

27. Sharma OP, Shah MV, Parikh DC, et al. Formulation optimization of gastroretentive drug delivery system for allopurinol using experimental design. Expert Opinion on Drug Delivery 2015;12(4):513-24.

28. Lee JH, Park TG, Choi H. Development of oral drug delivery using floating microspheres. J Microencapsul. 1999;16(6):715-29.

29. Nacht S, Katz M. The microsponge: A novel topical programmable delivery system. Drugs and the Pharmaceutical Sciences. 1990;42:299-325.

30. Osmani RA, Aloorkar NH, Kulkarni AS, et al. A new cornucopia in topical drug delivery: Microsponge technology. Asian J Pharm Sci Technol. 2014;4:48-60.

31. Dwivedi AM. Residual solvent analysis in pharmaceuticals. Pharmaceutical Technology. 2002;26(11):42-7. 
PICTORIAL ABSTRACT

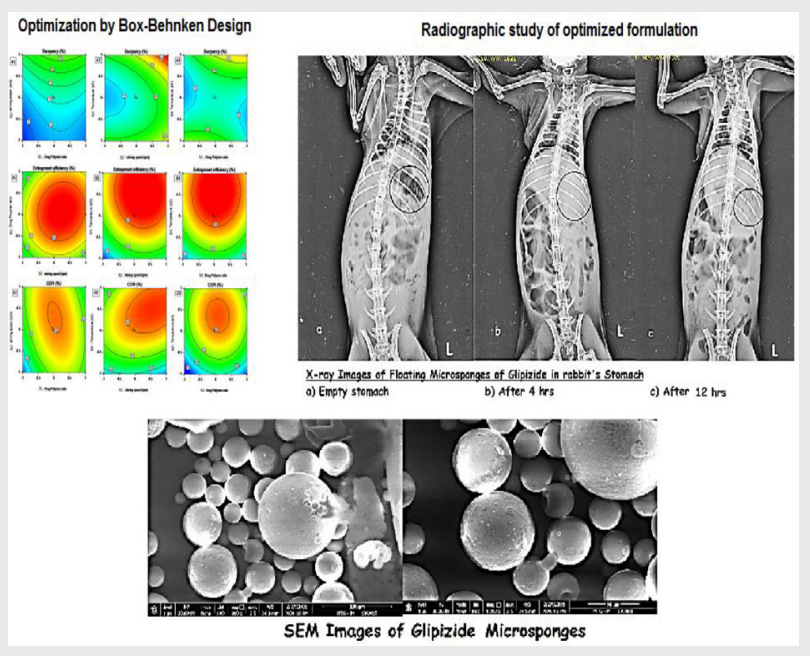

\section{SUMMARY}

The gastroretentive microsponges of Glipizide, an antidiabetic drug, were prepared by quasi-emulsion solvent diffusion method. The screening of critical formulation and process related Variables was done by Plackett-Burman Design and final optimization was done by Box-Behnken Design. The developed formulation can offer the sustained release of the drug at its site of absorption which may provide the better control of the diabetes due to less fluctuation in plasma drug concentration.

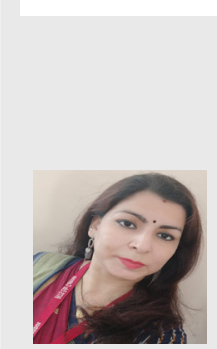

\begin{abstract}
About Authors
Meenakshi Bhavesh Patel (M. Pharm, PhD) is working as Associate Professor in Krishna School of Pharmacy \& Research, (KPGU), Krishna Edu Campus, Vadodara, Gujarat, India. She has teaching experience of more than 15 years. She has received the research grants from many government organizations. Her research areas of interest are: Formulation development, gastroretentive DDS, in-situ gelling system, solubility enhancement, etc.
\end{abstract}

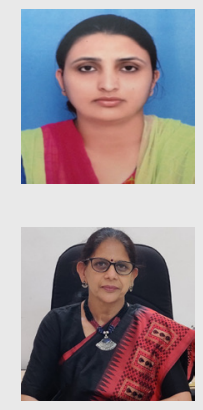

Farhatjahan Shaikh has received a Bachelor's and Master's degree from Sardar Patel University and a Doctorate Degree in Pharmaceutical Sciences from Gujarat Technological University in Gujarat, India. She has worked as an academician and researcher for more than 10 years. Her areas of expertise are formulation development, optimization and analytical method validation for pharmaceuticals.

Vandana B. Patel (M. Pharm, PhD, M.B.A. (HR)) has teaching experience of around 30 years. She has guided 55 PG students and $5 \mathrm{Ph}$. D. students for their dissertation. She is recipient of many prestigious awards namely: Shiksha Gaurav Puraskar Award in 2015, Innovation in Higher Education Award 2015, GTU Innovation Council - i2i (Innovate to Impact) Award, Bharat Excellence Award 2016, Best Researcher Award 2019, Principal of the Year Award 2019, APTI Women of the Year Award 2019, Iron Lady Samrasta Award 2019, 'Educational Leader' (SYMACHARYA Award 2020) and Academician of the Year Award (Academic Brilliance Awards-2020. Her research areas of interest are: Formulation development and optimization, solubility enhancement, analytical method development and validation etc. More than 120 number of full research papers and conference proceedings are published in International and National levels journals, under her guidance

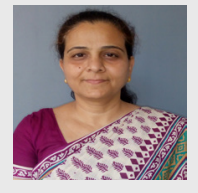

Naazneen Surti is ex-vice Principal and Professor, Babaria Institute of Pharmacy, BITS Edu Campus, Vadodara. She has 24 years of professional experience which is a blend of industrial experience and academia. She has guided more than 40 students for their PG dissertations, two students for PhD, 6 industry defined projects and currently supervising one PhD project. She has applied for 6 Indian patents, out of which 2 have been granted. She has authored 5 book chapters and 34 research publications in national and international journals of repute.

Cite this article: Patel MB, Shaikh F, Patel V, Surti N. Application of Experiential Design for Framing Gastroretentive Microsponges of Glipizide: Screening of Critical Variables by Plackett-Burman Design and Optimization by BoxBehnken Design. Indian J of Pharmaceutical Education and Research. 2021;55(4):966-78. 